\title{
Die Auswirkungen von Gewinn- und Entscheidungsbeteiligung auf den Unternehmenserfolg
}

\author{
Kornelius Kraft
}

Angenommen: 4. Februar 2011 / Online publiziert: 3. März 2011

(C) Institut für Arbeitsmarkt- und Berufsforschung 2011

Zusammenfassung Dieser Artikel bietet einen Überblick zu aktuellen Forschungsarbeiten hinsichtlich der Auswirkungen von organisatorischen Änderungen wie Gewinnoder Entscheidungsbeteiligung von Arbeitnehmern auf den Unternehmenserfolg. Die verwendete Methodik zur Berücksichtigung von möglichen Selektionsproblemen wird ausführlich erläutert. Dieser Rahmen wird zur Untersuchung der Effekte von freiwilligen Maßnahmen insbesondere Gewinnbeteiligung und gesetzlichen Vorgaben über das Betriebsverfassungs- und das Mitbestimmungsgesetz angewendet.

Schlüsselwörter Gewinnbeteiligung · Gesetzliche Mitbestimmung · Unternehmenserfolg .

Selektionsprobleme $\cdot$ Evaluationsmethodik

JEL Klassifikationen J53 · J54 · L25

The effects of profit sharing and worker participation on firm performance

Abstract This article summarizes recent studies concerning the effects of organizational change on fundamental economic variables. The framework applied to take account of possible selectivity effects is explained in detail. This methodology is applied to the effects of profits sharing and

K. Kraft ( $\square)$

TU Dortmund, Dortmund, Deutschland

e-mail: kornelius.kraft@uni-dortmund.de

K. Kraft

IZA, Bonn, Deutschland

K. Kraft

ZEW, Mannheim, Deutschland the legal provisions with respect to worker codetermination rights on the plant and firm level.

\section{Einleitung}

Organisationen unterliegen externen und internen Veränderungen. Aufgrund wandelnder Rahmenbedingungen oder neu zur Verfügung stehender Informationen kann es für Unternehmen sinnvoll sein, bestimmte organisatorische Maßnahmen einzuführen. $\mathrm{Zu}$ denken ist hierbei einerseits an nicht-monetäre Organisationssysteme wie größere Autonomie von Arbeitsgruppen, Delegation von Entscheidungsrechten oder das betriebliche Vorschlagswesen. Dadurch werden Arbeitnehmer stärker an das Unternehmen gebunden und es wird erwartet, dass die betriebliche Effizienz verbessert wird. Dieser Neuaufbau von Organisationen mit einer größeren Beteiligung der Arbeitnehmer hat in den letzten Jahren stark zugenommen. Andererseits rücken monetäre Anreize in den Vordergrund. Hierbei ist an Gewinn- oder Kapitalbeteiligung als kollektive Anreizsysteme zu denken.

Organisationen sind aber auch exogenen Einflüssen unterworfen. An dieser Stelle sind etwa gesetzliche Rahmenbedingungen zu nennen. Während endogene Änderungen normalerweise das Ziel haben, die Leistungsfähigkeit der Organisation zu erhöhen, können gesetzliche Vorgaben ganz andere Intentionen verfolgen. Bei politischen Entscheidungsprozessen spielen viele Faktoren eine Rolle, bei denen die Effizienzwirkung einer unter mehreren ist.

Bei den gesetzlichen Restriktionen werden das Betriebsverfassungsgesetz (BVG) und das Mitbestimmungsgesetz (MitbesG) betrachtet. Diese Gesetze regeln die Mitspracherechte der Arbeitnehmer und haben im In- und Ausland weite Beachtung gefunden. Das deutsche System der betrieblichen und der Arbeitnehmermitbestimmung ist weit ausge- 
baut und findet in anderen Ländern keine Parallele. Untersucht werden die Auswirkungen der Mitbestimmungsorgane auf zentrale ökonomische Größen sowie auch die Determinanten der Einführung.

$\mathrm{Ob}$ und in welchem Maße organisatorische Änderungen einen Einfluss auf verschiedene Größen wie z.B. Produktivität und Beschäftigung ausüben, wird im Rahmen des DFG-Projektes „Auswirkungen organisatorischer Änderungen auf den Unternehmenserfolg" unter Nutzung verschiedener Datensätze und moderner ökonometrischer Verfahren von 2004 bis 2010 untersucht. Hierbei zeigt sich, dass Unternehmen und ihre Arbeitnehmer in vielerlei Hinsicht von diesen Neuerungen profitieren können, die unternehmensspezifischen Gegebenheiten aber in Bezug auf die Effekte eine große Rolle spielen. Unternehmen mit ganz bestimmten beobachtbaren und unbeobachtbaren Charakteristika neigen dazu, bestimmte Organisationsstrukturen einzusetzen. Nur unter Berücksichtigung dieser Selektionseffekte ist es möglich, aus den Ergebnissen empirischer Studien klare Politikimplikationen abzuleiten.

\section{Das Selektionsproblem und verwendete Methoden}

Die bei Projektbeginn vorliegenden empirischen Untersuchungen basierten meistens auf Vergleichen zwischen Beobachtungen zu einem bestimmten Zeitpunkt. Die Beobachtungen sind typischerweise Unternehmen aber auch Arbeitnehmer, die sich hinsichtlich wichtiger Faktoren stark unterscheiden können.

Die Schätzung mit Hilfe von Querschnittsdaten ohne die Untersuchungseinheiten hinsichtlich der beobachteten Unterschiede zu bereinigen, lässt es allerdings schwierig werden, die kausale Beziehung zwischen den zentralen Variablen zu identifizieren. Diese Daten erlauben eigentlich nur die Bestimmung von Korrelationen, wobei die konkrete Wirkungsrichtung zwischen den untersuchten Größen aber weitgehend unklar bleibt. Jegliche Interpretation der beobachteten Korrelationen ist mit großer Unsicherheit verbunden. Dieses Problem wird aber von der Mehrzahl der Autoren ignoriert. Neben beobachtbaren Unterschieden zwischen Unternehmen bzw. Arbeitnehmern spielt auch unbeobachtbare Heterogenität eine Rolle, die bei vielen früheren Studien ebenfalls häufig nicht mit einbezogen wird.

Konkret auf das Projekt zu den Auswirkungen organisatorischer Änderungen wie Gewinn- und Entscheidungsbeteiligung bezogen heißt dies, dass beobachtbare und unbeobachtbare Unterschiede zwischen den Untersuchungseinheiten zum einen die Einführung neuer Organisationsmaßnahmen begründen, zum anderen aber auch die untersuchte Ergebnisvariable beeinflussen können. Heterogenität kann somit den Vergleich der Beobachtungen sehr erschweren, da unklar bleibt, inwiefern diese grundsätzlichen Unterschiede für die beobachteten Ergebnisse verantwortlich sind. Gerade Organisationsmaßnahmen werden vermutlich aus Überlegungen eingeführt, die betriebsspezifisch sind und dieselbe Maßnahme muss in einem anderen Betrieb durchaus nicht dieselbe Wirkung haben. Bei unzureichender Kontrolle impliziert dies die mögliche Existenz von Selektionsverzerrungen.

Ein monetäres Anreizsystem wie beispielsweise Gewinnbeteiligung wird aus bestimmten Gründen wie asymmetrischer Information oder unvollkommen beobachtbarem individuellem Output eingeführt. Die Vorteile können bei solchen Rahmenbedingen die möglichen Nachteile wie Trittbrettfahren durch schlecht motivierte Arbeitnehmer überwiegen. In anderen Firmen mag das nicht der Fall sein. Gefundene Effekte, wie z.B. eine höhere Produktivität, können dann nicht verallgemeinert werden. Sie werden dort gemessen, wo bestimmte Bedingungen vorliegen und die Betriebsleitung sich dann für die Einführung eines solchen Systems entscheidet. Sind diese Voraussetzungen nicht gegeben, so kann das Ergebnis ganz anders ausfallen. Dies wird bei reinen Querschnittsanalysen in der Regel vernachlässigt und die Ergebnisse müssen nicht automatisch auf einen kausalen Zusammenhang hindeuten.

Der forschungsleitende Gedanke dieses Projektes ist es, die tatsächliche Wirkungskette genauer zu untersuchen. Hierzu wird unter anderem auf moderne Verfahren aus der Evaluationsökonometrie zurückgegriffen. Diese Verfahren sind weit entwickelt, aber die Anwendung bleibt auf relativ wenige Gebiete beschränkt. Insbesondere bei der Evaluation von Maßnahmen der aktiven Arbeitsmarktpolitik werden diese Methoden häufig eingesetzt.

Das Grundproblem der mikroökonomischen Analyse des Effektes einer Maßnahme ist es, dass die gleiche Untersuchungseinheit nicht in zwei verschiedenen Zuständen beobachtet werden kann. Entweder wird die Maßnahme eingesetzt oder nicht. Um aber einen kausalen Effekt bestimmen zu können, müsste man wissen, wie sich die interessierenden Kennziffern eines von organisatorischen Änderungen betroffenen Unternehmens ohne diese Organisationsstrukturen entwickelt hätten. Die Herausforderung für den Forscher ist es nun, für die Beobachtungen, die eine bestimmte organisatorische Änderung vornehmen, Kontrollbeobachtungen aus der Gruppe der Unternehmen ohne diese Neuerung zu finden, die praktisch identische Merkmale aufweisen. Da die Unternehmen mit einer organisatorischen Änderung logischerweise nicht zum selben Zeitpunkt mit sich selbst ohne die Verwendung der organisatorischen Maßnahme verglichen werden können, werden ähnliche Firmen mit praktisch identischen Charakteristika bis auf die hier entscheidende Variable „organisatorische Änderung“ als Vergleichsbeobachtungen heran gezogen.

Das Ziel des vorliegenden Forschungsprojektes ist es, mit den verfügbaren ökonometrischen Methoden -soweit 
es die Datenlage zulässt- eventuell verzerrende Einflüsse herauszufiltern. Beobachtbare Unterschiede zwischen Unternehmen können durch die passende Wahl der Kontrollgruppe bereits mit Hilfe von Querschnittsdaten berücksichtigt werden. Anhand verschiedener Merkmale der Beobachtungen mit einer Neuerung werden hierzu möglichst ähnliche Vergleichsbeobachtungen ohne diese Neuerung aus einem Pool von Firmen bzw. Personen ausgewählt. Diese Auswahl geschieht mithilfe des sogenannten „MatchingVerfahrens". ${ }^{1}$ Dabei kann sich die Suche nach Beobachtungen mit und ohne ein bestimmtes Organisationssystem mit möglichst ähnlichen Eigenschaften bei vielen relevanten Charakteristika als schwierig erweisen. Eine Alternative stellt deshalb das „Propensity-Score Matching“ dar, bei dem für alle Beobachtungen die Wahrscheinlichkeit, eine bestimmte Maßnahme einzuführen, geschätzt wird. Im Anschluss wird eine Kontrollgruppe gebildet, die eine möglichst ähnliche Wahrscheinlichkeit hat, eine organisatorische Änderung vorzunehmen. Der Erfolg des Matchingverfahrens lässt sich beispielsweise über Tests hinsichtlich der Beseitigung vorher messbarer Unterschiede zwischen den Mittelwerten der verwendeten Variablen überprüfen. Bei einem erfolgreichen Matchingverfahren sollten diese Differenzen nicht mehr vorhanden sein.

Möglich ist aber auch, dass überlegene Organisationsmaßnahmen z.B. speziell von solchen Managern eingeführt werden, die auch in anderer Hinsicht ein Unternehmen effektiver als andere führen. Ein reiner Querschnittsvergleich ignoriert, dass möglicherweise Effizienzunterschiede aus vielerlei Gründen vorliegen und Produktivitätsvorteile schon vor der Einführung einer bestimmten Maßnahme vorhanden sein können. Für den Forscher unbeobachtbare Unterschiede zwischen Untersuchungseinheiten wie die Fähigkeiten des Managements bedürfen zur adäquaten Berücksichtigung Paneldaten.

Idealerweise sollten also sowohl Querschnitts- als auch Zeitreihendaten zur Verfügung stehen und viele Veränderungen bei den entscheidenden Variablen beobachtet werden. Mit Paneldaten ist es möglich, neben der Bildung einer adäquaten Kontrollgruppe die zeitliche Entwicklung einzubeziehen, d.h. zusätzlich Vorher-Nachher Vergleiche durchzuführen. Die Veränderung im Zeitablauf wird dann zwischen den Beobachtungen (Betriebe, Unternehmen oder Individuen) mit bestimmten unterschiedlichen Charakteristika verglichen (,Differenz-in-Differenzen-Schätzer“). Unbeobachtbare, konstant bleibende Eigenschaften können somit herausgefiltert werden.

Mit der Kombination der beschriebenen Methoden ist es also möglich, sowohl beobachtete als auch unbeobachtete

${ }^{1}$ Für eine detaillierte Beschreibung der verwendeten Evaluationsmethoden s. Heckman et al. (1999) oder Blundell und Costa Dias (2009).
Heterogenität zu berücksichtigen. Das Ziel dieser Vorgehensweise ist es, eine mögliche Verzerrung der Schätzergebnisse durch diese Heterogenität zu verhindern.

\section{Stand der Literatur}

Seit den achtziger Jahren werden die Auswirkungen von Gewinnbeteiligung relativ häufig untersucht. In diesem Zusammenhang gibt es widersprüchliche Anreize. Einerseits soll eine flexible Entlohnung hohe Leistung belohnen, andererseits wird das Ergebnis einer hohen Anstrengung mit allen anderen Mitarbeitern geteilt ( $1 / n$ Problem). Es ist deshalb unklar, ob sich Gewinnbeteiligung positiv auf die Leistungen der Arbeitnehmer auswirkt.

Im Vordergrund stehen deshalb Studien zur Produktivitätswirkung einer Gewinnbeteiligung, für die sich in der Regel positive Effekte feststellen lassen. ${ }^{2}$ Weniger häufig werden die Konsequenzen für Gewinne und die Fluktuation untersucht (vgl. z.B. Azfar und Danninger 2001). Auch für diese Faktoren finden sich für Beteiligungsunternehmen überlegene Ergebnisse.

Auf der Basis der fast einhellig positiven Effekte ist es erstaunlich, dass in praktisch allen Ländern ${ }^{3}$ nur eine Minderheit aller Firmen solche Systeme verwenden (Poutsma 2001). Diese Frage wurde bislang nicht überzeugend beantwortet.

Ebenfalls seit den achtziger Jahren werden die Effekte der Existenz von Betriebsräten untersucht. Betriebsräte werden auf Initiative der Belegschaft eingerichtet und insbesondere in kleineren und mittleren Betrieben finden sich keine Betriebsräte.

Die Vergleiche zwischen Firmen mit und ohne Betriebsräte führen nicht stets zu denselben Resultaten. Während die ersten Untersuchungen (FitzRoy und Kraft 1985, 1987a) eher pessimistische Einschätzungen gaben, kamen spätere Studien zu positiveren Ergebnissen (Addison et al. 2001). Ein wesentlicher Untersuchungsgegenstand ist auch in diesem Zusammenhang die Produktivität. Häufig werden sehr hohe Produktivitätsvorteile der Firmen mit Betriebsräten geschätzt. ${ }^{4}$ Auch in diesem Zusammenhang kann die kritische

\footnotetext{
${ }^{2}$ Die Vielzahl der Studien macht es wenig sinnvoll, alle hier aufzuführen. Im Folgenden sollen repräsentative Beispiele wie auch viel zitierte und richtungsweisende Arbeiten aufgeführt werden. Hierbei ist zu denken an: FitzRoy und Kraft (1987a), Hübler (1995), Möller (2000), Bellmann und Möller $(2005,2010)$ für Deutschland; Wadhwani und Wall (1990), Kruse (1992), Cahuc und Dormont (1997), Blasi et al. (2008) für andere Länder. Bei den meisten dieser Studien werden, teilweise auch aufgrund der Datenlage, keine Selektionseffekte berücksichtigt.

${ }^{3}$ Eine Ausnahme stellt Frankreich dar.

${ }^{4}$ So finden Frick (2002) und Frick und Möller (2003) einen positiven Effekt des Betriebsrates auf die Wertschöpfung, Jirjahn (2003) und Wagner (2008) kommen zu dem Ergebnis, dass die Tarifbindung in die-
} 
Frage gestellt werden, wieso sich angesichts der scheinbar vorhandenen Effizienzvorteile viele Firmen gegen Betriebsräte wehren. Anstatt die Produktivitätsvorteile kausal zu verursachen, könnten Betriebsräte auch dort bevorzugt eingerichtet werden, wo eine effektive Interessenvertretung der Arbeitnehmer besonders lohnend ist. Als Alternative zur Existenz von Betriebsräten könnten Veränderungen (Einrichtung eines Betriebsrates) und die damit verbundenen Wirkungen analysiert werden. Das ist natürlich auch kein triviales Unterfangen, da die Einrichtung eines Betriebsrates ein selten beobachtetes Ereignis ist. Somit wird eine große Grundgesamtheit an Beobachtungen benötigt, um eine ausreichende Anzahl an Betriebsrateinführungen zu erfassen. Bislang gibt es nur wenige Studien, die die Veränderungen nach einer Einführung betrachten.

Weniger intensiv als die Wirkungen der Existenz eines Betriebsrats wird die Unternehmensmitbestimmung erforscht. Auch hier war eine frühe Studie von FitzRoy und Kraft (1993) eher pessimistisch bezüglich der Produktivitätswirkungen. Die Untersuchung von Gorton und Schmid (2004) weist auf negative Effekte der paritätischen Mitbestimmung im Vergleich zur Drittelmitbestimmung auf die Rentabilität hin. Fauver und Fuerst (2006) zeichnen mit einer größeren Datengrundlage ein differenzierteres Bild. Andere Untersuchungen kommen ebenfalls zu weniger skeptischen Einschätzungen in Hinblick auf Produktivität und Innovation (FitzRoy und Kraft 2005, Kraft et al. 2010).

\section{Eigene Untersuchungen zu organisatorischen Änderungen}

Das Projekt befasst mit den Effekten verschiedener Organisationsstrukturen, wobei neben der Einteilung in monetäre und nicht-monetäre Organisationssysteme auch zwischen freiwillig von der Unternehmensleitung initiierten Maßnahmen und gesetzlich garantierten Eingriffen unterschieden werden kann.

Freiwillige Organisationsänderungen Bei den freiwilligen Maßnahmen steht in unserer Untersuchung die Gewinnbeteiligung im Vordergrund. Das Unternehmen wird eine Beteiligung dann realisieren, wenn der damit verbundene Verzicht auf einen Gewinnanteil langfristig durch eine höhere Arbeitsleistung, d.h. Produktivität ausgeglichen oder überkompensiert wird. Der Verzicht auf einen Anteil am Gewinn kann bei höherer Leistungsbereitschaft auch für den Unternehmer zu einem höheren absoluten Gewinn führen. Empirisch fanden in der Vergangenheit diverse Studien im Vergleich zu anderen Firmen eine höhere Produktivität dort vor, wo Gewinnbeteiligung eingesetzt wird.

sem Zusammenhang eine Rolle spielt. Addison et al. (2006) hingegen finden bei ihrer Analyse heraus, dass vom Betriebsrat keine positiven Produktivitätseffekte ausgehen.
Wie oben schon ausgeführt, stellt sich im Licht dieser eindeutigen empirischen Ergebnisse die Frage, wieso vergleichsweise wenig Unternehmen auf dieses flexible Anreizsystem setzen. Die Möglichkeit des Vorhandenseins spezieller Vorteile bei der Verwendung dieses Systems oder auch die denkbare Erklärung, dass die Firmen mit Gewinnbeteiligung grundsätzlich die besser geführten sind, wird in der Regel nicht untersucht.

Der Hypothese der Existenz von unternehmensspezifischen Vorteilen beim Einsatz bestimmter Organisationsformen wird mit Hilfe von modernen Evaluationstechniken nachgegangen. Die Datengrundlage bildet in diesem $\mathrm{Zu}-$ sammenhang das IAB-Betriebspanel, welches seit 1993 umfangreiche Informationen auf Betriebsebene erhebt. Unter anderem wird ein Vorher-Nachher Vergleich vorgenommen, bei welchem der Produktivitätsfortschritt im Zeitablauf gemessen wird. Es erweist sich, dass ein Großteil der beobachteten Produktivitätsunterschiede schon vorher vorhanden ist (Kraft und Lang 2010). Die besser geführten Firmen mit einem auch ohne Gewinnbeteiligung spürbaren Produktivitätsvorsprung entscheiden sich für eine Einführung von Gewinnbeteiligung. Die Gewinnbeteiligung erhöht zusätzlich die Leistung der betrachteten Firmen, aber ein reiner Querschnittsvergleich überschätzt die möglichen Produktivitätsgewinne.

Die genaue Identifikation der Auswirkungen von bestimmten Maßnahmen hat auch Politikimplikationen. In Frankreich ist es für Firmen mit mehr als 50 Beschäftigten Pflicht, ein Gewinnbeteiligungssystem einzuführen. In Großbritannien gibt es steuerliche Anreize für solche kollektiven Anreizsysteme. Auch in Deutschland werden steuerliche Begünstigungen für monetäre Formen der Mitarbeiterbeteiligung diskutiert und teilweise umgesetzt (wie bei der Mitarbeiterkapitalbeteiligung). Alle diese Maßnahmen basieren auf der Annahme, dass hierdurch positive Effekte für die beteiligten Firmen realisiert werden können. Unsere empirische Untersuchung zeigt, dass diese Vorteile überschätzt werden. Vermutlich ist die Einführung eines neuen Anreizsystems mit Fixkosten verbunden. Es ist deshalb fraglich, ob alle oder die meisten Firmen zu einer Einführung von solch einem Entlohnungssystem veranlasst werden sollten.

Es ist aber in jedem Fall ein Produktivitätseffekt der Einführung des Gewinnbeteiligungssystems zu spüren. Um den genauen Weg dieses Vorteils zu erklären, wurde der Zusammenhang zwischen Gewinnbeteiligung und Fortbildungsmaßnahmen einerseits und dem Innovationserfolg andererseits wiederum mithilfe von Daten des IAB-Betriebspanels untersucht. Auch bei Verwendung von modernen Methoden der Evaluationstechnik konnte gezeigt werden, dass die Einführung eines Beteiligungssystems in einem Betrieb die Weiterbildungsintensität, d.h. den Anteil der Beschäftigten, die an Weiterbildung teilnehmen, erhöht (Kraft und Lang 2008a). Ebenso werden Prozessinnovationen angestoBen (Aerts und Kraft 2008). Beides sind Bausteine für eine 
höhere Produktivität und unser Projekt scheint in der Lage zu sein, den Weg zu der beobachteten höheren Leistungsfähigkeit teilweise im Detail erklären zu können.

Bei der Einführung eines Gewinnbeteiligungssystems wird ein Teil des Gewinns an die Belegschaft abgegeben. Bei rationalem und gewinnmaximierendem Verhalten der Eigner müsste sich langfristig der den Kapitalgebern verbleibende Residualgewinn dennoch erhöhen. Dies ist möglich, wenn sich die Produktivität entsprechend erhöht.

Kraft und Ugarkovic (2006a) untersuchen die Auswirkung der Einführung einer Gewinnbeteiligung auf den Gewinnanteil, der den Kapitaleignern zukommt. Sie zeigen, dass dieser Gewinn im Durchschnitt steigt und sich somit diese Maßnahme für die Kapitalgeber lohnt. ${ }^{5}$

Ein anderes häufig verwendetes Anreizsystem ist das betriebliche Vorschlagswesen. Systeme dieser Art werden von vielen Firmen dazu eingesetzt, um die Mitarbeiter zu Beiträgen hinsichtlich technischer Verbesserungsmöglichkeiten anzuregen. Die Mitarbeiter, die eine bestimmte Technologie täglich verwenden, erwerben bei der konkreten Handhabung Wissen, worüber die Vorgesetzten nicht oder nur sehr eingeschränkt verfügen. Diese Ressource soll über ein monetäres Anreizsystem für die Organisationsoptimierung genutzt werden. Neben monetären Anreizmechanismen gibt es auch nicht-monetäre Systeme wie Gruppenarbeit oder Innovationszirkel, die den Innovationserfolg beeinflussen können und sollen. Trotz der weiten Verbreitung der aufgeführten Maßnahmen gab es bislang wenig repräsentative empirische Evidenz zu den Wirkungen solcher Systeme.

Mit Hilfe des Mannheimer Innovationspanels wurde untersucht, wie sich die Existenz eines betrieblichen Vorschlagswesens auf den Innovationserfolg auswirkt (Czarnitzki und Kraft 2008). Diese Systeme sind mit einer besseren Bilanz bei den Prozess- und Produktinnovationen verbunden. Durch den Einsatz betrieblicher Vorschlagswesen werden tatsächlich die erwünschten Ziele erreicht. Weiterhin hat die Delegation von Entscheidungen auf niedrigere Hierarchiestufen einen kostensenkenden Effekt und neue Organisationsformen können dazu beitragen, den aufgrund von Qualitätsverbesserungen erzielten Umsatz auszuweiten.

Aufgrund der Datenlage war es nicht möglich, bei dieser Fragestellung Selektionseffekte zu berücksichtigen. Da andere Untersuchungen im Rahmen des Projektes zeigen, dass unbeobachtbare Unterschiede zwischen den Unternehmen eine wesentliche Rolle für die Ergebnisse spielen können, sollten die Effekte eines betrieblichen Vorschlagwesens

\footnotetext{
${ }^{5}$ Die alternative Erklärung für steigende Gewinne wäre eine Substitution von laufenden Lohnzahlungen durch variable Gewinnanteile. Dies ist für Deutschland und andere entwickelte Industrieländer wohl eher selten der Fall, da sich die Arbeitnehmer und ihre Vertreter vermutlich gegen jede Lohnsenkung wehren würden. Es ist aber durchaus realistisch, dass etwa in Transformationsökonomien mit der Aussicht auf künftige Gewinnbeteiligung heutige Lohnkosten eingespart werden.
}

bei Verbesserung der Datenlage Untersuchungsgegenstand weiterer Studien sein.

Organisationsänderungen aufgrund gesetzlicher Vorgaben In einem weiteren Projektteil lag der Fokus auf den Konsequenzen von gesetzlichen Eingriffen. Wesentlich war auch hier, moderne Methoden zu verwenden, um die Kausalität der Abläufe, so gut wie dies möglich ist, zu identifizieren.

Die Mitbestimmungsregelungen in Deutschland lassen sich in die betriebliche Mitbestimmung und die Unternehmensmitbestimmung untergliedern. Die betriebliche Komponente umfasst Mitspracherechte, welche im Betriebsverfassungsgesetz festgelegt und über Betriebsräte ausgeübt werden. Die Unternehmensmitbestimmung bezieht sich auf das Mitbestimmungsgesetz, welches Mitbestimmung im Aufsichtsrat bei größeren Unternehmen vorschreibt.

Die Arbeit von Kraft und Lang (2008b) wendet sich den Ursachen für die Einführung von Betriebsräten zu. Betriebsräte können auf Initiative der Belegschaft eingeführt werden. Viele, insbesondere kleinere und mittlere Betriebe haben keinen Betriebsrat. Vermutlich erfolgt die Einführung eines Betriebsrates nicht zufällig, sondern dann wenn bestimmte Bedingungen vorliegen. Potentielle Gründe für die Einführung sind einerseits die Vorstellung, hierdurch die Verhandlungsmacht der Arbeitnehmerseite zu stärken und einen größeren Anteil am Unternehmenserfolg zu erhalten. Dies würde vermutlich insbesondere dann ein Grund sein, wenn es besonders viel zu verteilen gibt, d.h. die Stärkung der Verhandlungsmacht „lohnt“ sich für die Belegschaft.

Andererseits könnten Befürchtungen hinsichtlich der Arbeitsplatzsicherheit zu einer Einführung von Betriebsräten führen. Über das Betriebsverfassungsgesetz erhalten die Betriebsräte qualifizierte Möglichkeiten, bei notwendigen Entlassungen Verbesserungen für die betroffenen Arbeitnehmer zu erreichen. Folglich könnten Betriebsräte auch dann eingeführt werden, wenn die Belegschaft eine Verschlechterung der wirtschaftlichen Situation befürchtet. Jirjahn (2009) zum Beispiel kommt auch empirisch zu dem Ergebnis, dass Betriebsräte eher in wirtschaftlich schlechten Zeiten einführt werden.

Die beiden ausgeführten möglichen Erklärungen für eine Einführung eines Betriebsrates widersprechen sich grundsätzlich. Einen Anreiz zur Einführung könnte es geben, wenn es den betroffenen Betrieben besonders gut oder besonders schlecht geht.

Kraft und Lang (2008b) verwenden Daten des Sozioökonomischen Panels (SOEP). Diese Datenquelle befragt regelmäßig Haushalte zu vielen verschiedenen Schwerpunkten. Eine Frage bezieht sich auf die Beschäftigungssicherheit. Da das SOEP ein Panel ist, bei welchem nach Möglichkeit dieselben Personen interviewt werden, lässt sich auch hier ein Vorher-Nachher-Vergleich durchführen. Dies ist deshalb interessant, weil auch in Erfahrung gebracht 
wird, ob eine Person in einem Betrieb mit Betriebsrat tätig ist. Hierüber lässt sich auch die Veränderung dieser Situation erfassen, konkret ob zwischen zwei Perioden ein Betriebsrat eingerichtet worden ist.

Kraft und Lang (2008b) untersuchen, ob Sorgen über die Arbeitsplatzsicherheit die Wahrscheinlichkeit beeinflussen, dass ein Betriebsrat eingeführt wird. Auf diese Weise können wir Erwartungen als eine Determinante bei der Einführung eines Betriebsrates aufnehmen. Es erweist sich, dass Beschäftigte, die sich Sorgen um ihre berufliche Zukunft machen, häufiger in Betrieben arbeiten, die in späteren Perioden einen Betriebsrat eingerichtet haben. Betriebsräte werden demnach verstärkt dann nachgefragt, wenn die Beschäftigungssituation als kritisch eingeschätzt wird. Nach einer erfolgten Betriebsratseinführung gehen die Sorgen hinsichtlich der Beschäftigungssicherheit zurück. Entsprechend dieser Ergebnisse ist die Einführung eines Betriebsrates nicht in Verteilungsaspekten begründet, sondern erklärt sich aus den Befürchtungen, dass die Beschäftigung gefährdet ist. Ein Betriebsrat könnte in solchen Situationen für die Betroffenen die im Betriebsverfassungsgesetz vorgesehen Schutzmaßnahmen anwenden.

In einer weiteren Studie werden die Auswirkungen der Einführung von Betriebsräten auf das Beschäftigungswachstum untersucht. Frühere Studien beschränken sich größtenteils auf einen Vergleich zwischen Unternehmen mit und ohne einen Betriebsrat. Der reine Vergleich ist hinsichtlich der Identifikation von Ursache und Wirkung schlecht interpretierbar. ${ }^{6}$ Ist die Existenz ein Signal dafür, dass sich die Firma, in der der Betriebsrat vorgefunden wird, in einer kritischen ökonomischen Situation befindet? Will sich die Belegschaft mit Hilfe des BVG gegen ungerechtfertigte Entlassungen schützen? Oder finden sich Betriebsräte insbesondere in Firmen, die in ,alten“ Branchen angesiedelt sind und mit Strukturproblemen konfrontiert sind? Betriebsräte wären dann nicht ursächlich für eine schrumpfende Beschäftigung, sondern die Folge oder ein Indikator für gefährdete Branchen. Aber auch hier ist wieder die Situation denkbar, dass Betriebsräte eingeführt werden, um Renten abzuschöpfen. So hat die Belegschaft vielleicht in Erfahrung gebracht, dass der Betrieb sehr profitabel ist, während gleichzeitig keine Tariflöhne bezahlt werden.

Die Datengrundlage bildet hierbei wiederum das IABBetriebspanel. In diesem Zusammenhang wird festgestellt, dass die Einführung eines Betriebsrates mit verringerten Wachstumsraten bei der Beschäftigung einhergeht (Gralla und Kraft 2010a). Das reduzierte Beschäftigungswachstum

\footnotetext{
${ }^{6}$ Bisherige Analysen bezüglich der Effekte eines Betriebsrats auf das Beschäftigungswachstum führen zu keiner eindeutigen Schlussfolgerung. So kommen Addison und Teixera (2006) zu dem Ergebnis, dass ein Betriebsrat das Beschäftigungswachstum reduziert, Jirjahn (2008a, 2008b) hingegen findet mit einem anderen Datensatz und einer anderen Spezifikation der Unternehmensgröße keinen Effekt.
}

erklärt sich aus geringeren Einstellungs- aber nicht aus geringeren Entlassungsraten. Dieses Resultat geht mit dem vorherigen Ergebnis konform, dass verschlechterte wirtschaftliche Rahmenbedingungen die Einführung einer Arbeitnehmervertretung begünstigen. Alternativ hierzu kann aber die reduzierte Einstellungsquote auch aus den Erwartungen der Geschäftsleitung erklärt werden, dass die Entlassungskosten bei Existenz eines Betriebsrates steigen. Dies könnte schon vor einer möglichen Einstellung antizipiert werden, so dass einige Arbeitsplätze erst gar nicht besetzt werden und die Einstellungsrate zurückgeht.

Im Rahmen unseres Projektes wird die häufig geäußerte Vermutung untersucht, dass sich die Einführung der Mitbestimmung 1976 negativ auf die Eigenkapitalrendite auswirkt (Kraft und Ugarkovic 2006b). Zur Untersuchung dieser Frage verwenden wir Daten von 179 Unternehmen, für welche Angaben aus den 70er Jahren und später vorliegen. Konkret wird untersucht, ob die Einführung der Mitbestimmung die Eigenkapitalrendite der betroffenen Firmen reduziert hat. Damit Umwelteinflüsse und spezielle Gegebenheiten von mitbestimmten und nicht-mitbestimmten Unternehmen nicht das Ergebnis beeinflussen, werden die Veränderungen von mitbestimmten Unternehmen mit den Veränderungen von nicht-mitbestimmten Firmen verglichen. Der Vergleich von Wachstumsraten ermöglicht es, spezielle fixe Effekte und allgemein relevante zeitliche Veränderungen von den reinen Mitbestimmungseffekten zu trennen.

Für unser Sample findet sich keine Verschlechterung der Eigenkapitalrendite. Falls überhaupt eine Veränderung spürbar wird, so eher in Richtung auf eine Verbesserung. Die verbreitete Meinung, dass die Unternehmensmitbestimmung die Firmenrendite schmälert, findet durch unsere Studie keine Unterstützung.

Eine aktuelle Untersuchung befasst sich mit der konkreten Zusammensetzung des Aufsichtsrates und eventuell davon bestimmten Unterschieden bei Leistungsfaktoren (Gralla und Kraft 2010b). Konkret wird untersucht, wie sich die Existenz eines oder mehrer Großaktionäre auswirkt.

Untersuchungsgegenstand ist die Produktivität. Die Produktivität wird über die Schätzung einer Cobb-Douglas Produktionsfunktion geschätzt. Zusätzlich zu den Standardvariablen wird die Existenz eines oder mehrerer Großaktionäre berücksichtigt. Diese Variable wird mit der gesetzlichen Mitbestimmung interagiert.

Die Mitbestimmung hat in Firmen mit weit gestreuten Kapitalanteilen einen negativen Einfluss. Die Existenz von Großaktionären neutralisiert diesen Effekt jedoch. Ein gleichgerichtetes Ergebnis lässt sich für die Vertretung der Eigentümer im Aufsichtsrat zeigen. Ist ein Kapitaleigner in einem mitbestimmten Aufsichtsrat vertreten, so wirkt dies dem negativen Effekt der Mitbestimmung entgegen. 


\section{Fazit}

Ziel des Projektes war es, die Auswirkungen verschiedenster organisatorischer Änderungen auf unterschiedliche Unternehmenskennziffern zu untersuchen. Es zeigt sich, dass viele freiwillig eingeführte Maßnahmen wie Gewinnbeteiligung die erwarteten positiven Auswirkungen haben, aber auch von Unternehmen mit spezifischen Charakteristika eingeführt werden. Deshalb kann nicht automatisch davon ausgegangen werden, dass die gleichen Effekte auch für andere Unternehmen mit anderen Eigenschaften auftreten würden. Selektion scheint in Bezug auf Organisationssysteme eine maßgebliche Rolle zu spielen und muss berücksichtigt werden, um kausale Zusammenhänge aufdecken zu können und so eindeutige Politikaussagen zu ermöglichen.

Gesetzlich festgelegte Änderungen bezüglich der Mitbestimmung -wie die Einführung eines Betriebsrats- scheinen nicht, wie oftmals erwartet, negative Auswirkungen auf Unternehmen zu haben. Auch wenn das Beschäftigungswachstum nach Einführung eines Betriebsrats zurückgeht, muss das nicht die Folge dieser Maßnahme sein. Vielmehr scheinen Beschäftigte genau dann einen Betriebsrat einführen zu wollen, wenn sie eine Verschlechterung der Lage ihres Betriebs befürchten und sich damit auch um ihren Arbeitsplatz sorgen. Auch hinsichtlich der Unternehmensmitbestimmung finden sich nicht die negativen Effekte für ein Unternehmen, die in der Öffentlichkeit häufig unterstellt werden.

\section{Executive summary}

Organizations are frequently adjusted to changes in the economic environment. Such adjustments take place endogenously on an initiative of the owners, the management and/or the employees. Additionally organizations are affected by legal provisions. In particular the German codetermination rights grant employees considerable information, consultation and also veto rights.

This article reports the results of several empirical studies concerning the effects of organizational change on fundamental economic variables like productivity or employment. Earlier empirical studies are usually based on crosssectional data and the causal relation between the interesting variables remains unclear. One feature of our own studies is the application of modern evaluation methods to new topics. In particular matching and difference-in-differences are employed. By matching observable heterogeneity is taken into account. Matching eliminates differences between the observed characteristics of the treatment and control groups. Furthermore difference-in-differences takes account of unobserved heterogeneity. Additionally common time trends are allowed. Such methods are frequently implemented when active labour market policy is evaluated, but are much less frequently applied to other topics.
Concerning endogenous organizational measures firstly we consider the effects of profit-sharing. The productivity effects of profit-sharing have been analysed in a number of studies before and usually a positive impact is found. We distinguish between the productivity ahead of the introduction of a sharing system and the "true" productivity impact. It turns out that more than half of the observed productivity differences already exist before the system is introduced. However profit-sharing has a significant effect on performance. We also identify some of the basic reasons of the observed productivity advantages, as profit-sharing increases training intensity and process innovation.

Using a similar methodology, the project secondly considers the effects of the German system of codetermination rights. On the plant level works councils are powerful institutions representing workers interests. Works councils are not mandatory. They may be introduced, if the workers ask for such an institution. We find that works councils are adopted, in the case that employees suspect job losses.

On the plant level we find that works council reduce employment growth. This is mainly caused by lower hiring rates. There are rival explanations for this result: Works councils may not be the cause of lower hiring rates but the adoption may be a reaction to the suspected job losses. Alternatively works councils may increase (expected) dismissal cost and as this affects total labour costs employment is reduced.

If a company employs 2000 employees or more, on the supervisory boards the representatives of the employees gain access to $50 \%$ of the seats. The frequently suspected negative effect on profitability finds no support by our study. Codetermination on the supervisory boards does not reduce profits. Productivity may be unfavourably affected, but concentrated capital ownership neutralizes this negative effect.

Danksagung Der Deutschen Forschungsgemeinschaft (DFG) sei für die finanzielle Unterstützung des Projekts „Auswirkungen organisatorischer Änderungen auf den Unternehmenserfolg“ im Rahmen des Schwerpunktprogramms 1169 „Flexibilisierungspotentiale bei heterogenen Arbeitsmärkten“ herzlich gedankt.

\section{Literatur}

Addison, J.T., Schnabel, C., Schank, T., Wagner, J.: German works councils in the production process. Schmollers Jahrbuch 126, 251-283 (2006)

Addison, J.T., Schnabel, C., Wagner, J.: Works councils in Germany: their effects on firm performance. Oxf. Econ. Pap. 53, 659-694 (2001)

Addison, J.T., Teixera, P.: The effect of works councils on employment change. Ind. Relat. 45(1), 1-25 (2006)

Aerts, K., Kraft, K.: Profit sharing and innovation. Katholieke Universiteit Leuven, mimeo (2008)

Azfar, O., Danninger, S.: Profit-sharing, employment stability, and wage growth. Ind. Labor Relat. Rev. 54, 619-630 (2001)

Bellmann, L., Möller, I.: Produktivität, Fluktuation und Gewinnbeteiligung. In: Bellmann, L., Hübler, O., Meyer, W., Stephan, 
G. (Hrsg.) Institutionen, Löhne und Beschäftigung. Beiträge zur Arbeitsmarkt- und Berufsforschung, Bd. 294, S. 101-115. BeitrAB, Nürnberg (2005)

Bellmann, L., Möller, I.: Profit sharing and employment stability. Schmalenbach Business Rev. 62, 73-92 (2010)

Blasi, J.R., Freeman, R.B., Mackin, C., Kruse, D.L.: Creating a bigger pie? The effects of employee ownership, profit sharing, and stock options on workplace performance. NBER Working Papers: 14230 (2008)

Blundell, R., Costa Dias, M.: Alternative approaches to evaluation in empirical microeconomics. J. Hum. Resour. 44, 565-640 (2009)

Cahuc, P., Dormont, B.: Profit sharing: does it increase productivity and employment? A theoretical model and empirical evidence on french micro data. Labour Econ. 4, 293-319 (1997)

Czarnitzki, D., Kraft, K.: Mitarbeiteranreizsysteme und Innovationserfolg. Z. Arbeitsmarktforsch. 41(2/3), 245-258 (2008)

Fauver, L., Fuerst, M.E.: Does good corporate governance include employee representation? Evidence from German corporate boards. J. Financ. Econ. 82, 673-710 (2006)

FitzRoy, F., Kraft, K.: Unionization, wages, and efficiency: theories and evidence from the US and West Germany. Kyklos 38, 537554 (1985)

FitzRoy, F., Kraft, K.: Efficiency and internal organization: works councils in West German firms. Economica 54, 493-504 (1987a)

FitzRoy, F., Kraft, K.: Cooperation, productivity and profit sharing. Q. J. Econ. 102(1), 23-35 (1987b)

FitzRoy, F., Kraft, K.: Economic effects of codetermination. Scand. J. Econ. 95, 365-375 (1993)

FitzRoy, F., Kraft, K.: Co-determination, efficiency, and productivity. Br. J. Ind. Relat. 43(2), 233-248 (2005)

Frick, B.: Ökonomische Wirkungen der deutschen Betriebsverfassung. In: Sadowski, D., Walwei, U. (Hrsg.) Die ökonomische Analyse des Arbeitsrechts, S. 79-102. BeitrAB, Nürnberg (2002)

Frick, B., Möller, I.: Mandated works councils and firm performance: labor productivity and personnel turnover in German establishments. Schmollers Jahrbuch 123, 423-454 (2003)

Gorton, G., Schmid, F.: Capital, labour and the firm: A study of German codetermination. J. Eur. Econ. Assoc. 2, 863-905 (2004)

Gralla, R., Kraft, K.: Separating introduction- from selectivity-effects: the differences in employment patterns of co-determined firms. TU Dortmund, mimeo (2010a)

Gralla, R., Kraft, K.: Die Wirkung von Eigentümerkonzentration und Mitbestimmung auf die Produktivität. Erscheint in Schmollers Jahrbuch (2010b)

Heckman, J.J., Lalonde, R., Smith, J.: The economics and econometrics of active labor market programs. In: Ashenfelter, O., Card, D. (Hrsg.) Handbook of Labor Economics, Bd. 3, S. 1865-2097. Elsevier, Amsterdam (1999)
Hübler, O.: Produktivitätssteigerungen durch Mitarbeiterbeteiligung in Partnerschaftsunternehmen? In: Mitteilungen aus der Arbeitsmarkt- und Berufsforschung, Bd. 28, S. 214-223 (1995)

Jirjahn, U.: Produktivitätswirkungen betrieblicher Mitbestimmung Welchen Einfluss haben Betriebsgröße und Tarifbindung? Z. Betriebswirtsch. 73(4), 63-85 (2003)

Jirjahn, U.: Betriebsräte und Beschäftigungswachstum: Spielt die Spezifikation der Betriebsgröße eine Rolle für den geschätzten $\mathrm{Zu}$ sammenhang? Ind. Bezieh. 15(3), 279-291 (2008a)

Jirjahn, U.: Works councils and employment growth: a reply to Addison and Teixeira. Ind. Bezieh. 15(4), 436-448 (2008b)

Jirjahn, U.: The introduction of works councils in German establishments. Br. J. Ind. Relat. 43, 521-545 (2009)

Kraft, K., Lang, J.: Profit sharing and training. TU Dortmund, mimeo (2008a)

Kraft, K., Lang, J.: The causes and consequences of adopting a works council. In: Jahrbücher für Nationalökonomie und Statistik, Bd. 228/5+6, S. 512-532 (2008b)

Kraft, K., Lang, J.: Just a question of selection?-The causal effect of profit sharing on a firm's performance. TU Dortmund, mimeo (2010)

Kraft, K., Stank, J., Dewenter, R.: Co-determination and innovation. Camb. J. Econ. 35, 145-172 (2010)

Kraft, K., Ugarkovic, M.: Profit sharing and the financial performance of firms: evidence from Germany. Econ. Lett. 92, 333-338 (2006a)

Kraft, K., Ugarkovic, M.: Gesetzliche Mitbestimmung und Kapitalrendite. Jahrb. Natl.ökon. Stat. 226(5), 588-604 (2006b)

Kruse, D.L.: Profit sharing and productivity: microeconometric evidence from the United States. Econ. J. (Lond.) 102(410), 24-36 (1992)

Möller, I.: Produktivitätswirkung von Mitarbeiterbeteiligung. Mitt. Arb.markt- Berufsforsch. 32(4), 565-582 (2000)

Poutsma, E.: Recent trends in employee financial participation in the European Union. Luxembourg: Office for Official Publications of the European Commission (2001)

Wadhwani, S., Wall, M.: The effects of profit sharing on employment, wages, stock returns and productivity: evidence from UK micro data. Econ. J. (Lond.) 100(399), 1-17 (1990)

Wagner, J.: German works councils and producitvity: first evidence from a nonparametric test. Appl. Financ. Econ. Lett. 15, 727-730 (2008)

Kornelius Kraft Studium der Volkswirtschaftslehre an der Universität Heidelberg, Promotion zum Dr. rer. pol. 1984, Habilitation 1989 beides Universität Kassel, 1992-1993 o. Professor an der Universität Fribourg (CH), 1993-2003 Professor an der Universität Essen, seit 2003 Professor an der TU Dortmund.

Forschungsfelder: Arbeits- und Industrieökonomik. 